\title{
The Differences of Subjective findings in Pattern Recognition among Experts, Novices and Students: a Quasi-Delphi Technique
}

\author{
Kazuhisa Matsui ${ }^{1}$, Kotaro Kawaguchi ${ }^{2}$ \\ ${ }^{1}$ Department of Rehabilitation, Gifu Junior College of Health Science, ${ }^{2}$ Department of Physical Therapy, \\ School of Rehabilitation Science, Hyogo University of Health Sciences Japan.
}

$\begin{array}{ll}\text { ARTICLE INFO } \\ \text { Received } & : 26 / 06 / 2014 \\ \text { Accepted } & : 07 / 01 / 2015 \\ \text { Published } & : 25 / 03 / 2015\end{array}$

\section{KEYWORD}

Pattern recognition

Expert

Novice

Delphi technique

\begin{abstract}
Introduction: Experts use forward reasoning to diagnose accurately whereas non-experts prefer to use backward reasoning. Experts develop their hypotheses with pattern recognition before the interview and physical examination, using illness scripts for decision making and diagnosis. Objective: Superior pattern recognition is developed via clinical experience with patients. As a consequence, chunked subjective findings should be more abundant in experts than in novices. The purpose of this study was to clarify the differences in clinical picture between experts and novices in terms of subjective complaints that experts and non-experts look into and focus on when they initially see a patient. Method: Three rounds of questionnaires were administered using in a quasi-Delphi technique concerning two glenohumeral joint problems. Consensus was considered achieved when the distribution of answers had over $75 \%$ agreement in the scores. Result: 14 clinicians and 9 students were dropped out during 3 sequential surveys. The consensus was reached in three groups. At the final round, the findings of student group were integrated to 39 for frozen shoulder and 30 for rotator cuff tear. On the other hand, the findings in clinician group were converged to 34 and 36 for frozen shoulder and 28 and 35 findings for rotator cuff tear. Expert group demonstrated the specific example of aggravating factors and area of symptom as subjective findings. Conclusion: Consensus was achieved in all groups. Main difference between experts and the other groups were found in subjective findings.
\end{abstract}

(c) Medical Education Department, School of Medical Sciences, Universiti Sains Malaysia. All rights reserved.

CORRESPONDING AUTHOR: Kazuhisa Matsui, Department of Rehabilitation, Gifu Junior College of Health Sciences, Japan. Email: matsui@bz04.plala.or.jp

\section{Introduction}

Illness scripts, developed via various clinical experiences with new patients (1), are triggered unconsciously with patients' descriptions in most clinical reasoning processes used by experts (2) $(3,4)$. A randomized controlled study indicated improvement in diagnostic skills through the learning of illness scripts (4). On the other hand, no significant difference in diagnosis or treatment choice was demonstrated between medical students and clinicians even though the clinicians had more effective and accurate clinical reasoning processes (5).

Pattern recognition, the repertory of illness scripts to solve new problems by comparing and activating recall of similar clinical situations solved previously (2), is applied unconsciously within stored knowledge when clinical clues resemble what the clinician has experienced previously (6). The patterns can be seen in 
typical syndromes and tactics of management in addition to pathobiological and psychosocial factors (7). Understanding of pattern recognition is essential in successful management because patients with the same nominal pathology can have different contributing factors (7). The effectiveness of diagnosis using pattern recognition in contrast to using hypotheticodeductive reasoning has been stressed (2). This knowledge is applied to new patients in the form of forward reasoning. Differences of clinical reasoning processes between experts and novices have been reported by several authors. Experts use forward reasoning to diagnose accurately whereas nonexperts prefer to use backward reasoning $(8,10)$. Also, an expert has a sophisticated clinical picture to identify a patient's problem effectively on the basis of professional knowledge, professional craft knowledge, and personal knowledge. On the other hand, clinical reasoning beginners are inefficient in collecting information on patients and in finding their problems. Numerous forms of knowledge, elaborated causal networks, abridged networks, illness scripts and instance scripts are all characterized as belonging to an expert $(2,11)$.

Experts develop their hypotheses with pattern recognition before interviewing and physical examination using illness scripts for decision making and diagnosis (12). Our hypothesis was that superior pattern recognition was developed via clinical experience with patients. As a consequence, chunked subjective findings in experts would be more abundant than in novices. We thus expect pattern recognition to differ according to richness of clinical experience. The purpose of this study was to clarify differences in clinical picture between experts and novices in terms of subjective complaints that experts and non-experts look into and focus on when encountering a patient for the first time.

\section{Method}

\section{Participants}

Seventy-one experts on shoulder and musculoskeletal injuries (Japanese physiotherapists) participated as an expert panel whilst 91 (fourth year) undergraduate physiotherapy students in Japan who completed all of their lecture courses participated as a novice group (figure 1). The undergraduate students had already completed their coursework at school, including clinical rotations. Inclusion criteria for the panellists were experience and background regarding the topics, willingness to participate to achieve consensus and being an expert or having knowledge $(13,14)$. All panellists were eligible to participate in this study from the viewpoint of medical knowledge, although members of the novice group did not have well developed clinical reasoning skills. Workplaces of clinicians consisted of two clinics and eight hospitals. Students were studying in four universities.

\section{Procedure}

This study was approved by ethics committee in Hyogo University of Health Sciences. A pilot study for Delphi survey was conducted with respect to musculoskeletal disorders. Ten physiotherapy students and 21 musculoskeletal physiotherapists participated in an investigation on pattern recognition skills (15). Consensus of statements between non-experts was confirmed in a total of three rounds of a Delphi survey (15). Altogether three rounds of quasi-Delphi surveys were carried out to compare the pattern recognition between the two groups, clinicians and students. The clinician group was further divided into two subgroups, based on years of clinical experience: (i) one to five years of clinical experience (intermediate group) and (ii) six years or more of clinical experience (expert group) (figure 1).

The first session consisted of open queries on findings concerning adhesive capsulitis and rotator cuff tear, which would be influenced by how much the participants had seen patients with those problems. The answers were classified on the basis of disorder, subdivided further into subjective findings, objective findings, and contributing factors, and then listed for use in the second and third rounds. Similar responses were amalgamated into a form amenable to a Delphi style questionnaire. A five-step Likert scale (strongly disagree, disagree, undecided, agree 
and strongly agree) was used in the second and third rounds. Numerical responses (e.g., age), were transformed into quartile ranges for the second and third rounds.

In the second round, the panellists expressed degree of agreement using the Likert scale for each item generated by the initial questionnaire. For each item, the number of panellists involved in generating that statement was displayed, according to the Delphi method (13) (16).

In the final round, the participants expressed degree of agreement on responses of the second round. A final list was constructed from items for which consensus was achieved.

Each participant was asked to answer within ten days of receiving a questionnaire and required to refrain from discussing or studying any topic related to the questionnaires throughout the period of study, in order to prevent any external effects.

\section{Data analysis}

An item was deemed to have reached consensus if at least $75 \%$ of the panellists agreed or strongly agreed with it. This criterion can be used regarding convergence of opinions in the second and third rounds according to previous Delphi studies (17). To determine consensus of a numerical item, a range extending one quartile above and below the median of the third round data was used.

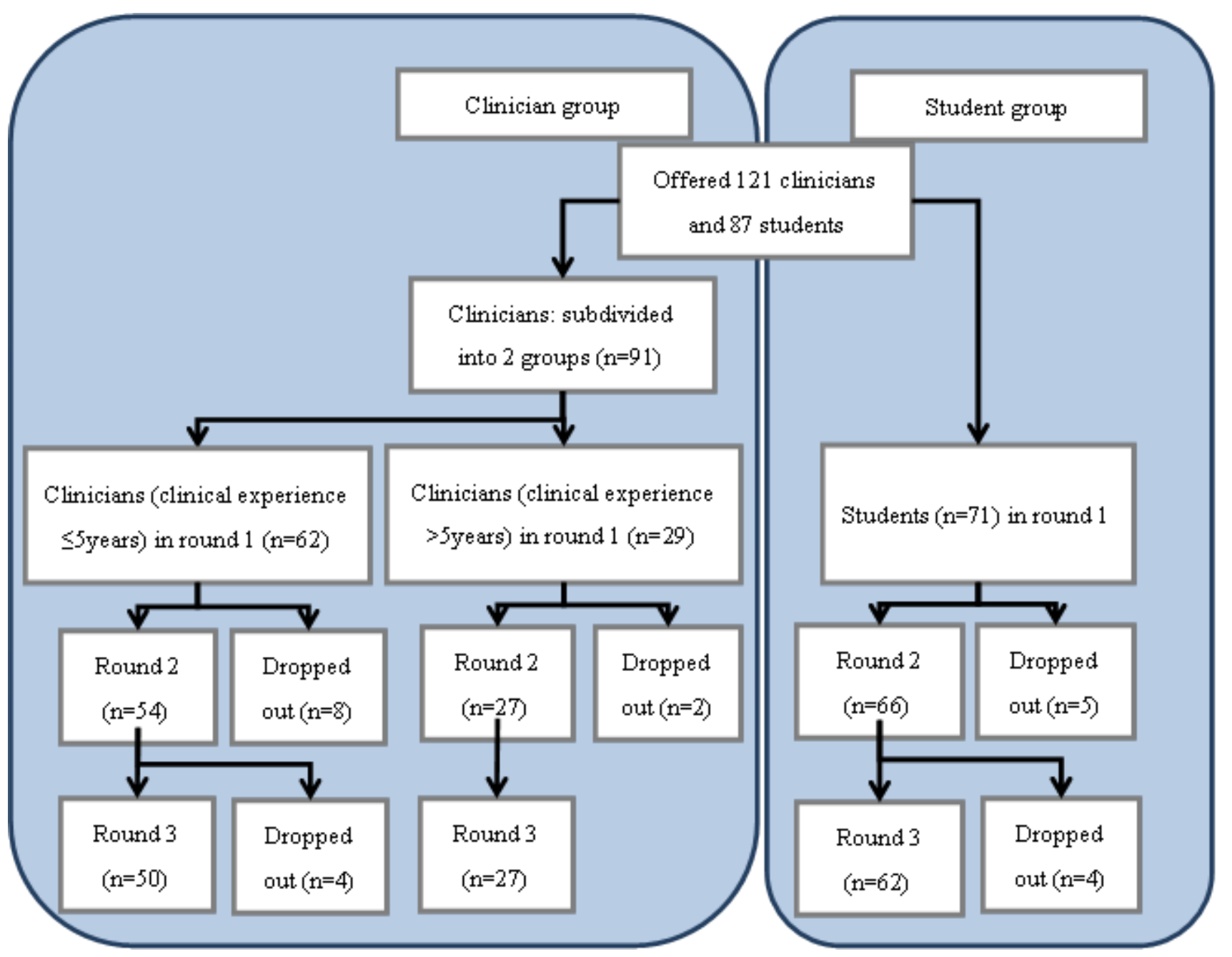

Figure 1: Flow chart of three rounds of surveys. 


\section{Result}

The response rate in the final round was $100 \%$ for all groups. Fourteen clinicians and nine students dropped out in the course of the three rounds (figure 1). Consensus was achieved in three groups. In the first round, the number of findings generated was higher in accordance with clinical experience (figure 2). Whereas the consensus of subjective findings was achieved sequentially through the second and third rounds in the group with more than five years of clinical experience, the students and the clinicians with up to five years of experience showed little change from the second to the third round (figure 2). In the first round, the student group generated 193 items for frozen shoulder and 157 items for rotator cuff tear, while intermediate and expert clinician groups made 269 and 229 items, respectively, for frozen shoulder and 238 and 213 items for rotator cuff tear. In the final round, the findings of the student group were narrowed down to 34 for frozen shoulder and 30 for rotator cuff tear. Findings in the intermediate and expert clinician groups converged to 34 and 36, respectively, for frozen shoulder and to 28 and 35 findings for rotator cuff tear.

The students and the intermediate clinicians showed poor examples for areas of symptoms and aggravating factors as subjective findings (table 1). Differences in subjective findings between frozen shoulder and rotator cuff tear were vague in these two groups. On the other hand, the expert group gave specific examples of aggravating factors and areas of symptoms (table 1). Whereas the students used textbook descriptions of symptoms, the two experienced groups described symptoms as patients would (table 1).

Table 1: subjective findings of frozen shoulder (top) and rotator cuff tear (bottom).

\begin{tabular}{lcc} 
Students & & \\
\multicolumn{1}{c}{ Subjective findings } & \multicolumn{2}{c}{ \% of consensus } \\
Round 2 & Round 3 \\
\hline Painful if moving shoulder & 99.35 & 97.89 \\
Pain during flexion & 85.38 & 89.80 \\
Pain during abduction & 79.31 & 93.08 \\
Pain if reaching an object on high place & 90.81 & 93.13 \\
Pain around end of range & 92.78 & 96.48 \\
Difficult to raise own arm & 89.43 & 97.29 \\
Impissible to raise own arm & 82.61 & 90.44 \\
Difficult to moving arm & 85.99 & 90.44 \\
Difficult to put own hand behind head & 95.04 & 92.02 \\
Difficult to put own hand behind back & 89.89 & 89.53 \\
Pain around shoulder & 94.46 & 85.94
\end{tabular}

Students

\begin{tabular}{lcc} 
Students & \multicolumn{2}{c}{ \% of consensus } \\
\multicolumn{1}{c}{ Subjective findings } & 98.28 & 95.64 \\
Pain over shoulder & 83.14 & 90.80 \\
Discomfort on shoulder & 94.44 & 93.63 \\
Pain if shoulder abducted/ rotated externally & 96.90 & 95.57 \\
Pain if moving shoulder & 95.64 & 92.37 \\
Difficult to move shoulder & 88.93 & 85.77 \\
Pain if raising own arm & 81.75 & 82.28 \\
Feeling of weakness & 93.55 & 94.40 \\
Difficult to raise own arm & 95.76 & 96.92 \\
Impossible to raise own arm & 85.77 & 90.40 \\
Difficult to put own hand behind head & 87.88 & 87.95
\end{tabular}

\begin{tabular}{lcc} 
Clinicians ( $\mathbf{5}$ 5y clinical experience) \\
\multicolumn{1}{c}{ Subjective findings } & \multicolumn{3}{c}{$\%$ of cons ensus } \\
Round 2 & Round 3 \\
\hline $\begin{array}{l}\text { Pain over anterior aspect of shoulder } \\
\text { Painful if moving shoulder }\end{array}$ & 89.19 & 89.55 \\
Night pain & 98.75 & 86.80 \\
Impossible to lying on the painful side & 88.05 & 89.39 \\
Difficult to changing clothes & 88.84 & 86.29 \\
Impossible to raise own arm & 82.86 & 89.23 \\
Impossible to raise own arm due to pain & 86.55 & 86.73 \\
Difficult to raise own arm & 89.24 & 91.00 \\
Pain if reaching to high place & 90.58 & 87.88 \\
& 87.91 & 88.00 \\
Difficult to put own hand behind back & 90.67 & 84.85 \\
Pain if putting own hand behind back & 88.07 & 86.22 \\
Difficult to putting on clothes & 89.30 & 83.25 \\
Pain when changing clothes & 87.91 & 82.81 \\
Difficult to washing own hair & 87.68 & 81.96
\end{tabular}

Clinicians ( 55 y clinical experience)

\begin{tabular}{ccc} 
Clinicians ( $\mathbf{5}$ y clinical experience) & \multicolumn{2}{c}{$\begin{array}{c}\text { \% of cons ensus } \\
\text { Subjective findings }\end{array}$} \\
Round 2 & Round 3
\end{tabular}

$\begin{array}{lll}\text { Omalgia } & 95.81 & 90.00\end{array}$

Pain if moving shoulder

Impossible to raise own arm

Difficult to raise own arm

Feeling of weakness

Pain if raising own arm

Pain if raise own arm laterally

Pain if taking something on high place

\begin{tabular}{lcc} 
Clinicians (>5y clinical experience) & & \\
\multicolumn{1}{c}{ Subjective findings } & \% of consensus \\
& Round 2 & Round 3 \\
Pain over shoulder & 92.79 & 91.51 \\
Vague pain shoulder to upper arm & 78.00 & 88.46 \\
Painful if moving shoulder & 97.22 & 97.27 \\
Night pain & 94.74 & 83.02 \\
Difficult to raise own arm & 94.54 & 100.00 \\
Trouble with reaching forward & 82.69 & 88.46 \\
Impossible to put own hand behind back & 94.74 & 94.55 \\
Difficult to wash own hair & 88.46 & 94.34 \\
Difficult to changing clothes & 88.24 & 88.46 \\
& & \\
Pain at end of range & 84.31 & 88.00 \\
& & \\
Impossible to raise own arm & 84.62 & 90.20 \\
Aching over shoulder & 100.00 & 94.55 \\
Impossible to raise own arm & 94.44 & 83.64 \\
Pain if putting own hand behind back & 88.46 & 83.33 \\
It is hard to take something of a high place & 94.23 & 88.89 \\
Impossible to lying on the affected side & 68.75 & 83.02 \\
Pain if raising own arm & 94.44 & 83.33
\end{tabular}

Clinicians (>5y clinical experience)

\begin{tabular}{lcc}
\multicolumn{1}{c}{ Subjective findings } & \multicolumn{2}{c}{$\%$ of consensus } \\
Round 2 & Round 3 \\
Omalgia & 94.55 & 100.00 \\
Pain with elevation & 94.55 & 100.00 \\
Impossible to raise own arm & 90.57 & 88.46 \\
Impossible to hold the position during & 94.34 & 94.12 \\
tooth blushing/ using a blowdryer & & \\
Difficult to raise own arm laterally & 82.69 & 100.00 \\
Resting pain in acute stage & 75.00 & 81.63 \\
Pain in raise own arm laterally & 88.46 & 100.00 \\
Pain in combing & 68.09 & 81.63 \\
Pain when carrying a heavy object & 94.23 & 94.34 \\
Difficult to put own hand behind head/ & 88.46 & 88.00 \\
back & & \\
Pain with motion & 94.64 & 94.34
\end{tabular}



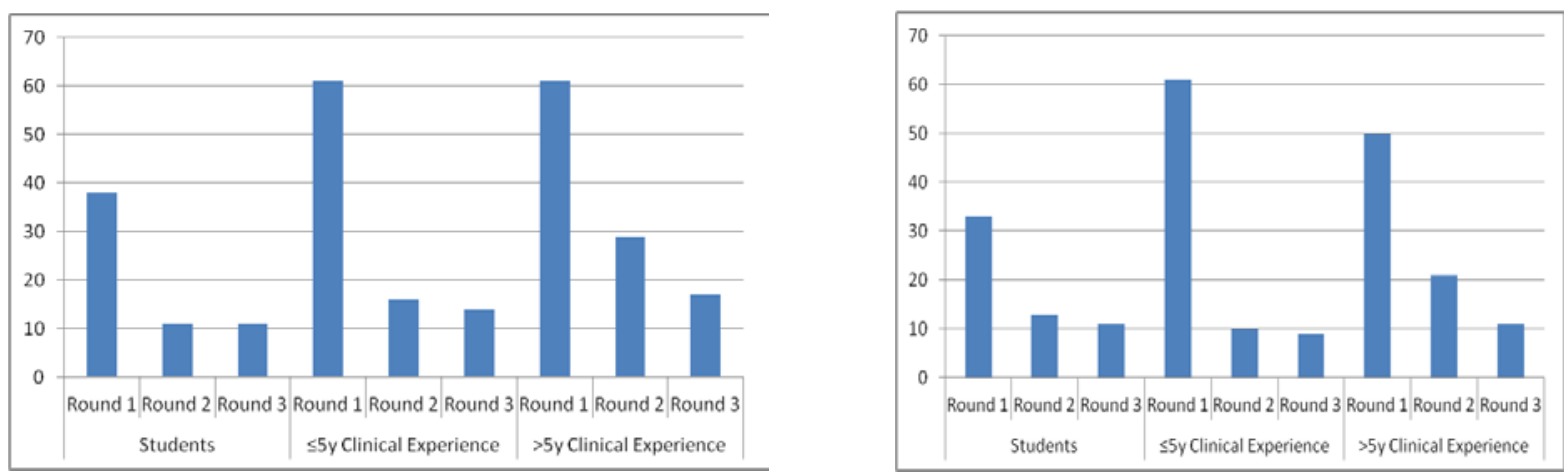

Figure 2: subjective findings for frozen shoulder (right) and in rotator cuff tear (left). The number of findings increased in proportion to clinical experience in round 1. The consensus of subjective findings were achieved gradually in those with more than five years of clinical experience whilst those of student and therapists with five or fewer years of clinical experience groups did not changed much between the second and third rounds.

\section{Discussion}

Major differences were found in subjective findings. The number of subjective findings was proportional to clinical experience (Figure 2). The difference between experts and the other groups was in knowing precisely what the aggravating factors were. This could be a consequence of clinical experience since illness scripts are applicable to new patients (1). Expert physiotherapists observe and interact with patients to finalize decision making whereas novice physiotherapists collect information from the medical record (18). Rich subjective findings by the experts might come from interaction with patients. Excellent problem description is a feature of experts $(3,19)$ and is activated when experts interview and conduct physical examinations (12). The process upon which experts effectively build their hypotheses and differentiate among possible disorders (20) might be based on these organized subjective findings. The importance of subjective and objective examinations has been advocated (21). Our hypothesis that clinical experience with patients develops pattern recognition ability was demonstrated, in contrast with a past study reporting that diagnostic accuracy between students and clinicians did not differ (3).

Illness description in the expert group appeared to be more concise. The expert groups pointed out relationships among pain, range of motion restriction and muscle weakness as objective findings in frozen shoulder while the descriptions of subjective findings in the student group were close to textbook explanations (table 1). Previous clinical experience helps clinicians collect information (22). Chunked knowledge from previous experience is integrated in experts (18). As a result, subjective findings in experts may be more sophisticated. Experts thus might better be able to interpret patients' words to elicit subjective complaints. On the other hand, pattern recognition by students is formed from a knowledge network (23). The reasoning process in students is worked out patient by patient (24). However, students were able to diagnose accurately if they happen to have obtained specific knowledge from study of the disease (25). Students thus describe what they know in the manner of a textbook (table 1). What is tested on physical examination might be decided according to what is known via subjective examination. Experts sift through various hypotheses whilst students pick up possibilities of problems in a reasoning process (19). The difference in pattern recognition between students and the expert groups might be a consequence of degree of prior experience with patients who have had similar problems. The matured pattern recognition develops accurate diagnosis and treatment strategy (26).

The panellists for the Delphi method have to be experts or knowledgeable (13) (14). However, panellists who had wide-ranging clinical experience but were not experts in 
musculoskeletal physiotherapy (i.e., students) participated in this study. The success of consensus being achieved among non-experts using the quasi-Delphi technique was demonstrated in our preliminary study (15). Thus, the illness scripts among the three groups were comparable. Being an expert is not simply a matter of clinical experience, as matured knowledge with sophisticated clinical reasoning is required (27). Nevertheless, the clinician groups were categorized for this study on the basis of clinical experience since pattern recognition was sophisticated throughout clinical experience (2) (28). A group composed of participants who solve problems in different ways and have diverse personalities show higher quality and adequacy of solutions (16). As this study aimed to clarify differences of pattern recognition between experts and novices rather than to elucidate clinical features of certain glenohumeral joint problems, the quasi-Delphi technique and the participants were appropriate for the methodology of this study. Thus, consensus could be achieved in all groups even although their results were not uniform. Students need to examine several cases of a given impairment to be able to attain diagnostic reliability since knowledge from only one case is inadequate for application to other cases, be they similar or dissimilar (28). This study investigated data related to pattern recognition abilities that the panellists possessed. Thus the influence of knowledge of specific clinical problems should not have had a great influence on the results.

The Delphi technique has been used to survey the clinical features of several musculoskeletal problems (29-31). However, the validity using the Delphi technique for clinical findings is controversial (32). This study elucidated differences in pattern recognition among the novice, intermediate and expert groups.

Two glenohumeral disorders were chosen to observe how experts and students use pattern recognition and how they differentiate these disorders from other glenohumeral problems while gathering findings from patients. The specific disorders were chosen according to previous research. Tendinitis, rotator cuff injury, impingement syndrome, frozen shoulder and osteoarthritis have been reported as the majority of shoulder disorders in mature patients who are referred from general clinics and have long histories of physiotherapy treatment $(21,33)$. Only two of the several clinical shoulder problems were chosen in this study to avoid unnecessary complexity in comparing consensus between novices and experts.

This study investigated how physiotherapists develop their own pattern recognition abilities. Physiotherapists with expertise in orthopaedic disorders and who had a wide range of clinical experience participated. Even orthopaedic physiotherapists who had one year of clinical experience participated. They might not yet have been experts on musculoskeletal disorders, although a strict relationship between clinical experience and clinical reasoning skills is questionable. The aim was to clarify differences in pattern recognition among students who hardly have any clinical experience, novice physiotherapists who have some clinical experience, and seasoned physiotherapists who have much clinical experience. The results demonstrated that the description of subjective complaints differed on the basis of clinical experience.

A limitation of this study might be that the comparison was only of pattern recognition by physiotherapists for musculoskeletal problems of the shoulder. The object was to study pattern recognition as a clinical reasoning process. If this survey had been on neurological problems or with other medical professionals, the results might have been different. Another limitation is that the time to prepare a response was not controlled because of distance factors. Significant correlation has been noted between diagnostic accuracy and immediate response time in written cases (34). The results might have been different if answering within a specific time constraint had been required. Such issues could be addressed in future studies.

\section{Conclusion}

Pattern recognition in both experts and novice 
groups was surveyed using a quasi-Delphi technique. Consensus achieved by each group represented the key features of two glenohumeral joint disorders. Students relied on textbook descriptions for interview content whilst experts responded to patients' words to infer subjective complaints.

\section{Acknowledgement}

I would like to express my gratitude to all panellists who were willing to participate in this study and to university faculty who helped organize this survey.

\section{Reference}

1. Charlin B, Tardif J, Boshuizen HPA. Scripts and medical diagnostic knowledge: theory and applications for clinical reasoning instruction and research. Academic Medicine. 2000; 75:182-90.

2. Coderre S, Mandin H, Harasym PH, Fick GH. Diagnostic reasoning strategy and diagnostic success. Medical Education. 2003; 37: 695-703.

3. Gilhooly KJ. Cognitive psychology and medical diagnosis. Applied Cognitive Psychology. 1990; 4: 261-272.

4. Lee. A, Joynt GM, Lee AKT, Ho AMH, Groves M, Vlantis AC et al. Using illness scripts to teach clinical reasoning skills to medical students. Family Medicine. 2010; 42(4): 255-261.

5. Monagemi A, Rikers RMJP, Schmidt HG. Clinical case processing: a diagnostic versus management focus. Medical Education. 2007; 42: 1166-1172.

6. Charlin B, Boshuizen HPA, Custgers EJ, Feltovich PJ. Scripts and clinical reasoning. Medical Education. 2007; 41: 1178-1184.

7. Jones MA, Rivett DA. Introduction to clinical reasoning. In: Jones MA, Rivett DA, editors. Clinical Reasoning for Manual Therapists. Edinburgh: Butterworth Heinemann; 2004. p. 3-24.

8. Coderre SP, Harasym P, Mandin H, Fick G. The impact of two multiple-choice question formats on the problem-solving strategies used by novices and experts. BMC Medical
Education. 2004; 4(23): [about 9 p.]. Available from: http://www.biomedcentral.com/content/pdf/ 1472-6920-4-23.pdf

9. Patel VL, Groen GJ. Knowledge based strategies in medical reasoning. Cognitive Science. 1986; 10: 91-116.

10. Rivett DA, Jones MA. Improving clinical reasoning in manual therapy', In: Jones MA, Rivett DA, editors. Clinical Reasoning for Manual Therapists. Edinburgh: Butterworth Heinemann; 2004. p. 403-419.

11. Schmidt HG, Norman GR, Boshuizen HPA. A cognitive perspective on medical expertise: theory and implications. Academic Medicine. 1990; 65(10): 611621.

12. Charlin B, Brailobsky C, Leduc C, Blouini $D$. The diagnosis script questionnaire: a new tool to assess a specific dimension of clinical competence. Advances in Health Science Education. 1998; 3: 51-58.

13. Hsu CC, Sandford BA. The Delphi technique: making sense of consensus. Practical Assessment, Research \& Evaluation. 2007; 12: 1-8.

14. Rowe G, Wright G. The Delphi technique as a forecasting tool: issues and analysis. International Journal of Forecasting. 1999; 15: 353-375.

15. Matsui K, Kawaguchi K. The development of pattern recognition via clinical experience: a preliminary study. Education in Medicine Journal. 2014; 6: e72-e80.

16. Powell C. The Delphi technique: myths and realities. Methodological issues in nursing research. 2003; 41: 376-382.

17. Carnes D, Mullinger B, Underwood $M$. Defining adverse events in manual therapies: A modified Delphi consensus study. Manual Therapy. 2010; 15: 2-6.

18. Wainwright SF, Shepard KF, Harman LB, Stephens J. Factors that influence the clinical decision making of novice and experienced physical therapists. Physical Therapy. 2010; 91: 87-101.

19. Bowen JL. Educational strategies to promote diagnostic reasoning. The New England Journal of Medicine. 2006; 355: 2217-2225. 
20. Johnson PE, Durán AS, Hassebrock FH, Moller J, Prietula M. Expertise and error in diagnostic reasoning. Cognitive Science. 1981; 5: 235-283.

21. Steavenson JH, Trojian T. Evaluation of shoulder pain. Journal of Family Practice. 2002; 51(7): 605-611.

22. Terry W, Higgs J. Educational programmes to develop clinical reasoning skills. Australian Physiotherapy. 1993; 39(1): 4751.

23. Boshuizen HPA, Schmidt HG. The development of clinical reasoning expertise. In: Higgs J, Jones MA, Loftus S, Christensen N, editors. Clinical reasoning in the health professions. $3^{\text {rd }}$ ed. Edinburgh: Butterworth Heinemann; 2008. p. 113-121.

24. Stone L. Reasoning for registrars: an overview for supervisors and medical educators. Australian Family Physician. 2008; 37(8): 650-653.

25. Auclair F. Problem formulation by medical students: an observation study. BMC Medical Education. 2007; 7 (16): [about 5 p.]. Available from: http://www.biomedcentral.com/content/pdf/ 1472-6920-7-16.pdf

26. Monajemi A, Schmidt HG, Rikers MJP. Assessing patient management plans of doctors and medical students: an illness script perspective. 2012; 32: 4-9.

27. Jones MA. Clinical reasoning process in manipulative therapy In: Boyling JD, Palastanga N, Jull GA, Lee DG, Grieve GP, editors, Grieve's Modern Manual Therapy. $2^{\text {nd }}$ edn, Edinburgh: Churchill Livingstone; 1994. p. 471-489.

28. Rolfe IE, Sanson-Fisher RW. Translating learning principles into practice: a new strategy for learning clinical skills. Medical Education. 2002; 36: 345-352.

29. Cook C, Brismée JM, Sizer PS. Subjective and objective descriptors of clinical lumbar spine instability: A Delphi study. Manual Therapy. 2006; 11: 11-21.

30. Smart KM, Blake CB, Staines A, Doody C. Clinical indicators of 'nociceptive', 'peripheral neuropathic' and 'central' mechanisms of musculoskeleral pain. A Delphi survey of expert clinicians. Manual
Therapy. 2010; 15: 80-87.

31. Walmsley S, Rivett DA, Osmotherly PG. Adhesive capsulitis: establishing consensus on clinical identifiers for stage 1 using the Delphi technique. Physical Therapy. 2009; 89: 906-917.

32. Wilde VE, Ford JJ, McMeeken JM. Indicators of lumbar zygapophyseal joint pain: survey of an expert panel with the Delphi technique. Physical Therapy. 2007; 87: 1348-1361.

33. Ginn KA, Herbert RD, Khouw W, Lee R. A randomized controlled clinical trial of a treatment for shoulder pain. Physiocal Therapy. 1997; 77(8): 802-809.

34. Sherbino J, Dore KL, Wood TJ, Young ME, Gaissmaier W, Kreuger S et al. \& Norman, GR The relationship between response time and diagnostic accuracy. Academic Medicine. 2012; 87(6): 785-791. 